\title{
The radio continuum from a planetary nebula as a mirror of the central star evolution
}

\author{
Olga I. Sharova \\ Radio physical Research Institute, 25/12 Bolshaya Pecherskaya st., 603950 Nizhny Novgorod, \\ Russia \\ email: sho10358@yandex.ru
}

\begin{abstract}
In the frame of ISW model the rates of the evolutionary changes of the flux density and the critical frequency in radio continuum spectrum have been calculated in dependence on the kinematic age of a planetary nebula. The time changes of the instantaneous radio spectra of IC 418 and NGC 6369 do not contradict accounts.
\end{abstract}

Keywords. Planetary nebulae: general, radio continuum: ISM

The evolutionary changes of parameters of the central star should be reflected in changes of a spectrum of emission from a planetary nebula. Variations are found in lines of an optical range by Kostyakova(1999). In radio continuum the essential changes are found out from some very young planetary nebulae (see for example Knapp et al. 1995). A search of changeability is of great interest in a radio continuum from planetary nebulae at more late stages of evolution.

The theoretical thermal radio spectrum of a nebula with uniform density may be presented as function of frequency $\nu(\mathrm{GHz})$ :

$$
S_{\nu}=A \cdot y^{2}\left[0.5-y^{4.2}+\exp \left(-y^{-2.1}\right)\left(y^{2.1}+y^{4.2}\right)\right],
$$

where $y=\nu / \nu_{c}, \quad \nu_{c}$ is the critical frequency $(\mathrm{GHz})$,

Let's estimate rates of the evolutionary changes in a radio spectrum from nebula

$$
\delta S_{\nu}=\frac{100}{S_{\nu}} \cdot \frac{\Delta S_{\nu}}{\Delta t}\left(\% \cdot \mathrm{yr}^{-1}\right) \text { and } \delta \nu_{c}=\frac{100}{\nu_{c}} \cdot \frac{\Delta \nu_{c}}{\Delta t}\left(\% \cdot \mathrm{yr}^{-1}\right)
$$

in the framework of ISW model suggested by Kwok(1982) with velocities of stellar winds from a red giant and from a central star $5 \mathrm{~km} \mathrm{~s}^{-1}$ and $3000 \mathrm{~km} \mathrm{~s}^{-1}$ accordingly and a mass loss rate from a red giant $10^{-4} \mathrm{M}_{\odot} \mathrm{yr}^{-1}$. The parameters of the central stars are taken from paper by Blöcker (1995) as a function of an age of a star $t$.

The rates of change of flux density at $15 \mathrm{GHz}$ are shown in Fig. 1 depending on kinematic age of a nebula $\tau$ :

$\tau=\frac{R}{V_{S}}, \quad$ where $R=\left\{\begin{array}{ll}R_{0}, & R_{0}<V_{S} \cdot t \\ V_{S} \cdot t, & R_{0}>V_{S} \cdot t\end{array}, \quad R\right.$ is the observable radius of a nebulae, $R_{0}$ is the radius of a zone of ionization of hydrogen, $V_{S}$ is an expansion velocity.

On an interval $\tau \leqslant 100$ years there is a growth of a flux density for all models. After 100 years in model with $0.696 \mathrm{M}_{\odot}$ there is an interval of time with appreciable fall of a flux up to $5 \%$ per year. In other models the reduction of a flux occurs at later stages and does not exceed shares of percent per year.

The critical frequency strongly depends on the mass of star nucleus. For the nucleus mass $0.546 \mathrm{M}_{\odot}$ it is always lower than $1 \mathrm{GHz}$. It grows in models with masses of nucleus 

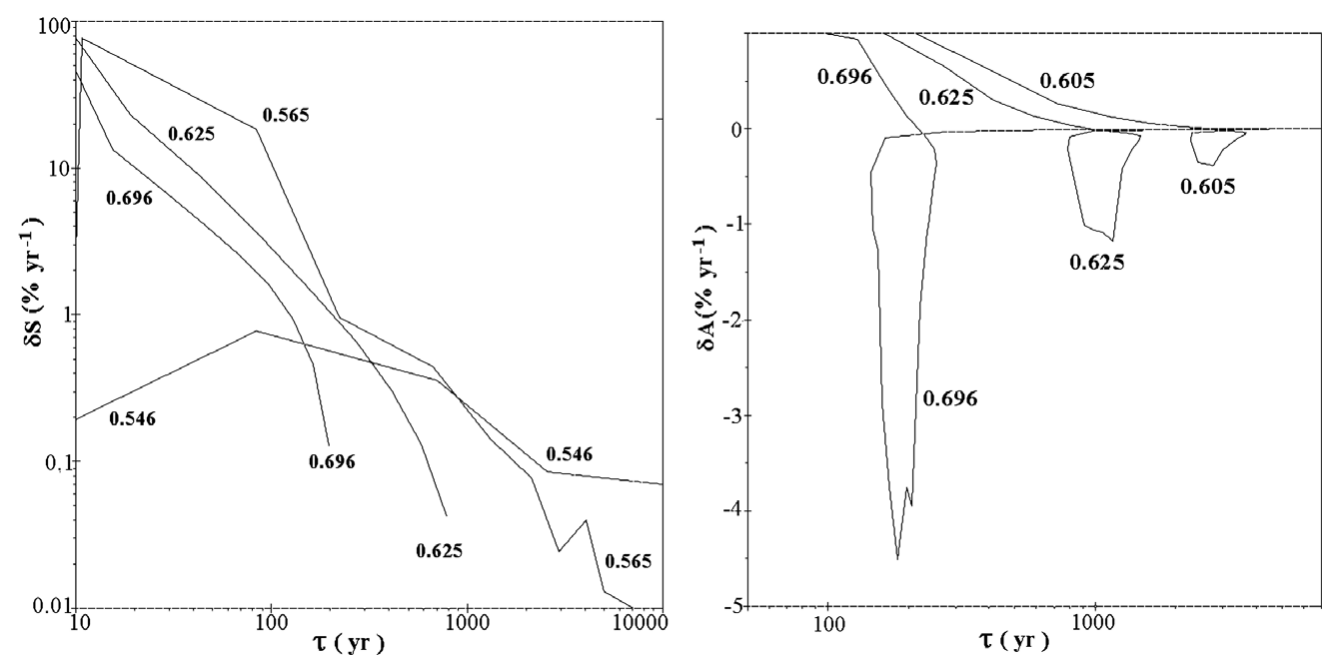

Figure 1. The rates of increasing (left) and decreasing (right) of the flux density $S_{15}$ at $15 \mathrm{GHz}$ as a function of the kinematic age $\tau$ of a nebula. The figures on lines correspond to masses of nucleuses of the central stars.

Table 1. Parameters of radio spectra of planetary nebulae

\begin{tabular}{|c|c|c|c|c|c|c|c|c|}
\hline \multirow{2}{*}{ Planetary nebula } & \multicolumn{3}{|c|}{ Epoch 1989.163} & \multicolumn{3}{|c|}{ Epoch 2002.333 } & \multirow{2}{*}{$\begin{array}{c}\delta \mathbf{S}_{15} \\
\left(\% \mathbf{y}^{-1}\right)\end{array}$} & \multirow{2}{*}{$\begin{array}{c}\delta \nu_{\mathbf{c}} \\
\left(\% \mathbf{y}^{-1}\right.\end{array}$} \\
\hline & $\nu_{\mathrm{c}}(\mathrm{GHz})$ & $\mathbf{A}(\mathbf{J} \mathbf{y})$ & $\mathrm{S}_{15}(\mathrm{Jy})$ & $\nu_{\mathbf{c}}(\mathbf{G H z})$ & A (Jy) & $\mathrm{S}_{15}(\mathrm{Jy})$ & & \\
\hline IC 418 & 1.71 & 6.43 & 1.72 & 1.76 & 5.38 & 1.43 & -1.0 & 0.2 \\
\hline NGC 6369 & 1.14 & 6.79 & 1.75 & 1.20 & 6.12 & 1.58 & -0.6 & 0.3 \\
\hline
\end{tabular}

$0.565 \mathrm{M}_{\odot}$ and $0.605 \mathrm{M}_{\odot}$ while $\tau \leqslant 1000$ years with rate not higher than $0.4 \%$ per year. In other models the critical frequency monotonously decreases.

The instantaneous spectra of two planetary nebulae were obtained in a wide range of frequencies including both limiting cases of optical thickness by using radiotelescope RATAN-600 SAO RAS. The measurements are carried out in February 1989 by Sharova (1999) at frequencies 0.96, 3.65, 3.95, 7.7, $11.2 \mathrm{GHz}$ and in April 2002 at 2.3, 3.95, 7.7, 11.2, 21.7 under the program offered by the author (Sharova 2011). The parameters of a thermal spectra were calculated by a method of the least squares and are shown in Table 1. The fitting of data by thermal spectra is good.

The found out changes in radio spectra do not contradict accounts, if the masses of the central stars are from 0.625 up to $0.696 \mathrm{M}_{\odot}$, and kinematic ages do not exceed 1500 years. There is an actual problem to study time variations of radio continuum from planetary nebulae by monitoring of the instantaneous spectra in wide interval of the frequencies.

\section{References}

Blöcker, T. 1995, A\&AA, 299, 755

Kostyakova, E. B. 1999, PAZ, 25, 457

Kwok, S. 1982, AJ, 258, 280

Knapp, G. R., Bowers, P. F., Young, K., \& Phillips, T. G. 1995, AJ, 455, 293

Sharova, O. I. 1999, Thesis

Sharova, O. I. Mingaliev, M. A., et al. 2011, in preparation 